\title{
Estimation of Intra- and Inter-ventricular Dyssynchronization with Cardiac Magnetic Resonance Imaging
}

\author{
O Grebe, A Mueller, N Merkle, J Wöhrle, L Binner, \\ M Höher, V Hombach, *H Neumann, ${ }^{\circ} \mathrm{HA}$ Kestler
}
Dept. of Cardiology, ${ }^{\circ}$ Forschungsdozentur Bioinformatik and
*Dept. of Neural Information Processing, University of Ulm, Ulm, Germany

\begin{abstract}
Aim of this study was to evaluate quantitative parameters for inter- and intra-ventricular dyssynchronicity measurement. 10 patients with severely reduced left ventricular function (LVF) and variable $Q R S$ duration (70\% had left bundle branch blockation; LBBB) and six healthy subjects (control) were examined with cine magnetic resonance imaging. The phase differences between the left and right ventricle were estimated from the endo- and epicardial contours of the full left and right ventricle in short axis slices for the whole cardiac cycle. Inter-ventricular asynchronicity (INTER) and Intraventricular asynchronicity (INTRA) were significantly higher in patients with reduced LVF as compared to the control group independently from the presence of a $\angle B B B$. INTRA but not INTER could discriminate patients with and without $\angle B B B$. We found two clinically very promising parameters describing the phase difference of the left and right ventricle.
\end{abstract}

\section{Introduction}

In patients with reduced left ventricular function (LVF) a concomitant left bundle branch block (LBBB) leads to a further deterioration of global heart performance due to asynchronous contraction. Implantation of biventricular pacemakers is an option for these patients [1]. QRS duration as a parameter for electrical synchronicity alone has failed to serve as a predictive marker for the clinical outcome of patients after biventricular pacing, since mechanical synchronicity does not necessarily require a synchronous electrical excitation [2]. Up to now there is lack of a quantitative parameters for inter- and intra-ventricular dyssynchronicity. In contrast to echocardiography, magnetic resonance imaging (MRI) offers an excellent discrimination of both left and right ventricular endocardial borders of the whole heart with a high spatial and temporal resolution.

Aim of this study was the evaluation of simple parameters for inter- and intra-ventricular dyssynchronization measurement derived from MRI.

\section{Methods}

\subsection{Subject data}

The patient group consisted of 10 patients with severely reduced left ventricular function (LVF) due to dilated cardiomyopathy (DCM). Left ventricular ejection fraction was median $29 \%$ (range $7 \%-41 \%$ ). QRS duration ranged from 97 to $220 \mathrm{~ms}$. $70 \%$ had left bundle branch blockation (LBBB). Median age was 66.5 years (range 42 to 71). The control group (control) comprised six healthy subjects without any heart disease Median EF 70\%, QRS duration shorter than $120 \mathrm{~ms}$ and median age 30 years.

\subsection{Imaging}

Imaging was performed on a $1.5 \mathrm{~T}$ whole body scanner (Intera CV, Philips Medical Systems) with Master Gradients (slew rate $150 \mathrm{~T} / \mathrm{m} / \mathrm{s}$, amplitude $30 \mathrm{mT} / \mathrm{m}$ ) and software Release 8.1. A 5-element phased-array cardiac coil was used. Three short survey scans were performed to define the position and true axis of the left ventricle. Afterwards, wall motion was imaged during breath holding with in long and short-axis slices using a steadystate free precession (balanced fast-field echo) sequence, which provides an excellent endocardial contrast. Cardiac synchronization was achieved by prospective gating. The cine images were recorded with 23 heart phases (23 frames per heart cycle).

\subsection{Image analysis}

As a basis to estimate the dyssynchronization, all endo-and epicardial borders of the full left and right ventricle were manually drawn on 10 to 11 short axis slices for the whole cardiac cycle using the MASS plus Software Release 5.0 (Medis, Leiden, The Netherlands). The papillary muscles were assigned to the ventricular cavum (see Fig. 1). The contour description was then imported into Matlab. 
After extracting the contours the phase differences between the left and right ventricle were estimated by the following two methods. Inter-ventricular asynchronicity (INTER): The diameters of both contours were determined via a graphic line search procedure along their centers of gravity. To reduce the noise in the phase estimation the line search was performed 11 times in 1.5 degree steps around these centers. These 11 geometric points were averaged to get a noise reduced estimate of the cutting points between the line connecting both centers and the two contours (see Fig. 2). For each slice the phase difference between the two time series describing the diameters of the two cardiac regions were computed. The time series were normalized to have mean zero and Euklidian norm one. The phase value was then computed as the arc cosine of the scalar product of the two normalized time vectors. Mean and variance over the phase values of the innermost five slices were evaluated. Intra-ventricular asynchronicity (INTRA): The phase difference of the septal and lateral border of the left ventricle to its mean center of gravity was used as an indicator of asymmetry in the movements of the left ventricle. The center was determined as the average point over all phases for each slice separately.

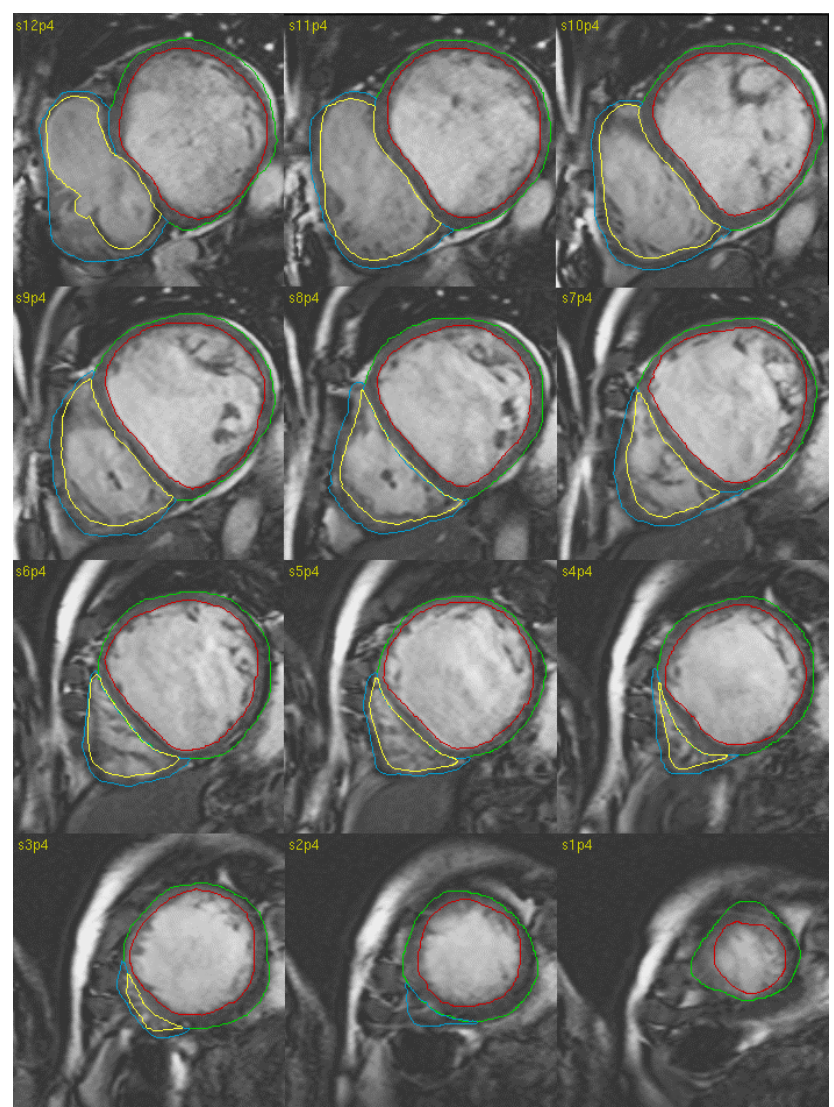

Figure 1. MR short axis slices acquired from a patient with dilated cardiomyopathy (note the flattened interventricular septum). The hand-drawn endocardial and epicardial contours are shown.

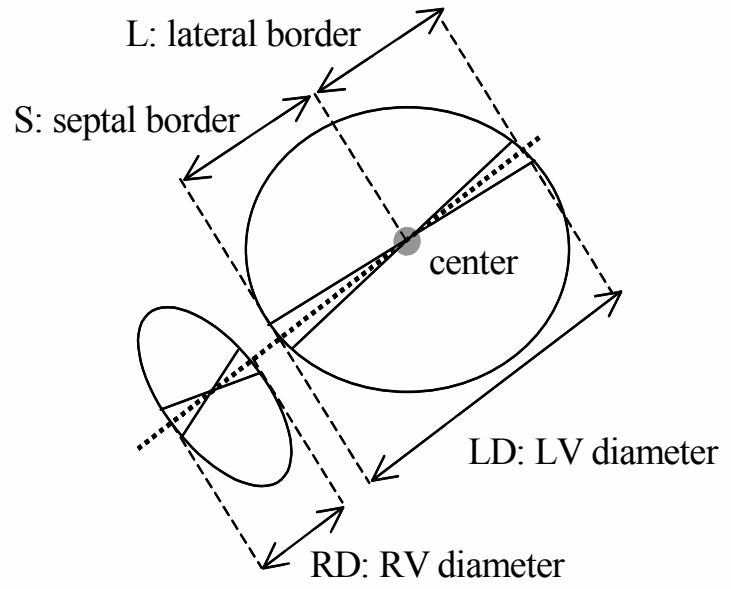

Figure 2. Illustration of INTER and INTRA. The orange line connects the two centers of gravity. INTER: The inter-ventricular phase is evaluated from the phase difference of the two time variant values $\mathrm{LD}$ and $\mathrm{RD}$ (bottom). INTRA: The intra-ventricular phase value computes to the phase difference of $\mathrm{S}$ and $\mathrm{L}$ which are the distances of the averaged center of gravity to the septal and the lateral border point (the dotted line intersecting the left ventricular contour).

\subsection{Statistics}

Continuous data are presented as median and interquartile ranges. For analysis of differences between groups, the Mann-Whitney U-test was used. A statistical probability of $\mathrm{p}<0.05$ was considered to be significant.

\section{Results}

Figure 3 illustrates the median for the two parameters. In the control group the median INTER was 0.47 and INTRA was 0.54 . Both parameters were significantly higher in patients with reduced LVF and LBBB (INTER 1.06, $\mathrm{p}=0.001$ and INTRA 1.84, $\mathrm{p}=0.001$, and without LBBB (INTER 1.01, $\mathrm{p}=0.048$ ) and INTRA, $\mathrm{p}=0.024$ ). INTRA but not INTER could discriminate patients with and without LBBB (INTRA $\mathrm{p}=0.017$, INTER $\mathrm{p}=0.667$ ).

In Figure 4 a correlation of the new parameters to the QRS duration is shown. The correlation was only significant for INTRA.

\section{Discussion}

Aim was the evaluation of the benefit of MRI for the characterization of mechanical asynchronicity in patients with reduced LVF. The striking advantage of MRI is the high, almost patient-independent image quality with the ability to image the whole left and right ventricle in short axis slices. A study using tissue doppler echocardiography [3] has demonstrated a good prediction for a functional recovery after resynchronization, however, a good ultrasound source and an experienced investigator are mandatory. 


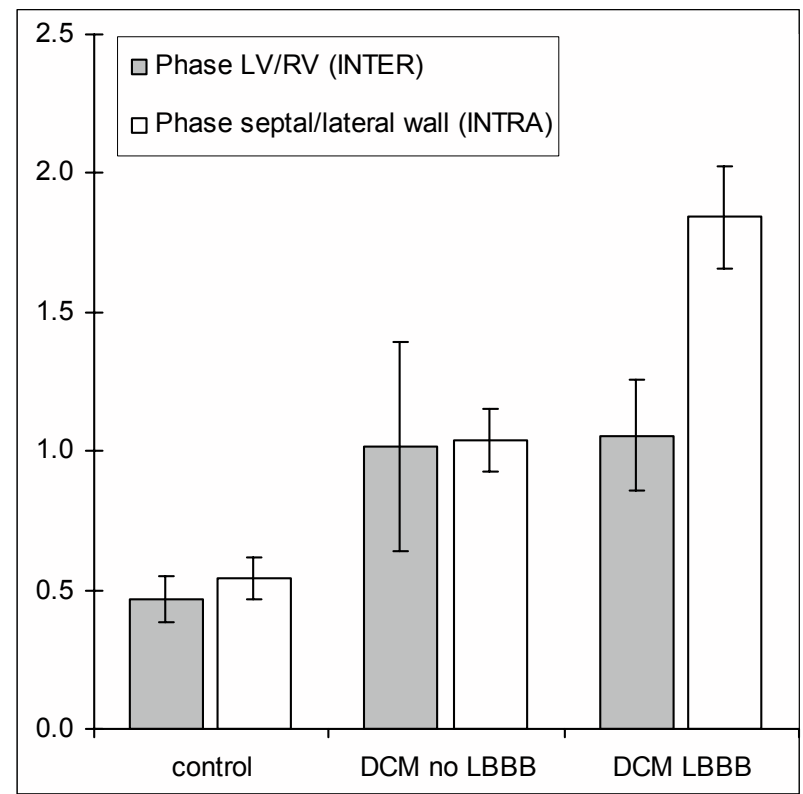

Figure 3. Median for the phase differences of the left and right ventricle (INTER) as well as the septal and lateral left ventricular wall contraction (INTRA). Both INTRA and INTER can discriminate between the control group and the DCM patients but only INTRA describes the patients with LBBB.

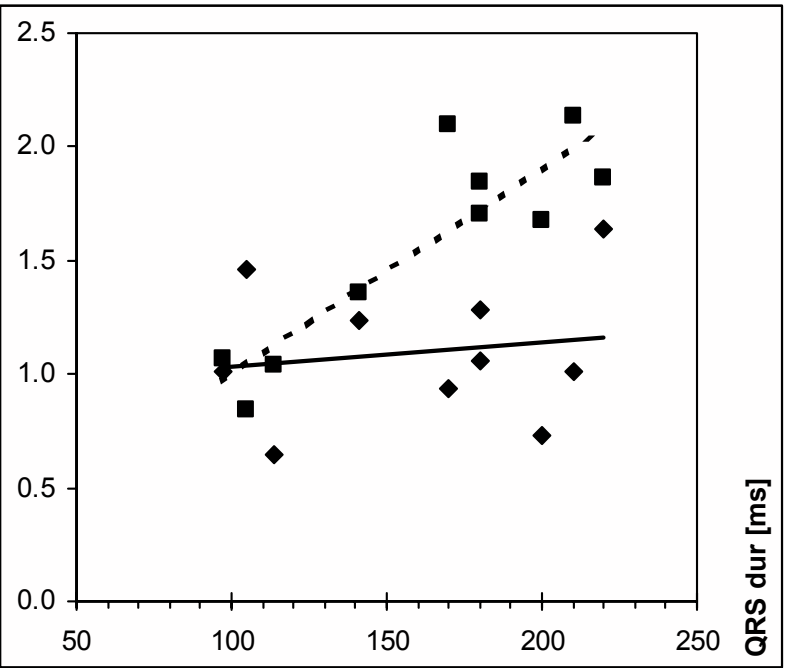

Figure 4. Correlation matrices of the QRS duration and the parameter INTRA (boxes and broken line, Pearson $\mathrm{r}=0.88$ ) and INTER (diamonds, $\mathrm{r}=0.15$ ). Only the correlation for QRS and INTRA was significant.
In this pilot study two parameters that characterize the mechanical inter- and intraventricular synchronicity were developed. INTER was found to be independent from the QRS duration and might serve as a new feature to characterize mechanical dyssynchronicity. Although the follow-up of these parameters with MRI is not feasible in patients after implantation of a pacemaker until MR-safe implants are available, it is possible to test the clinical and prognostic value in a prospective study. End point will be the recovery of the patient's exercise capacity.

A possible application can be to use 3D-ultrasound images instead of MRI, which has the advantage of a feasible follow-up during pacemaker stimulation. It still has to be demonstrated whether the quality of contours derived from ultrasound images provide similar results, has to be determined.

\section{Conclusion}

Two clinically very promising parameters describing the phase difference of the left and right ventricle and the contraction of the left ventricle were obtained. These parameters may be of special interest for the decision of implanting a biventricular pacemaker, which so far is based on QRS duration.

\section{References}

[1] Cazeau S, Leclercq C, Lavergne T et al. Effects of multisite biventricular pacing in patients with heart failure and intraventricular conduction delay. N Engl J Med 2001; 344:873-80

[2] Leclercq C, Faris O, Tunin R, Johnson J, Kato R, Evans F, Spinelli J, Halperin H, McVeigh E, Kass DA. Systolic improvement and mechanical resynchronization does not require electrical synchrony in the dilated failing heart with left bundle-branch block. Circulation 2000;106:1760-3

[3] Sogaard P, Egeblad H, Kim WY, Jensen HK, Pedersen AK, Kristensen BO, Mortensen PT. Tissue Doppler imaging predicts improved systolic performance and reversed left ventricular remodeling during long-term cardiac resynchronization therapy. J Am Coll Cardiol 2002;40:23-30 (2002)

Address for correspondence:

Olaf Grebe, M.D.

University of Ulm

Dept. of Cardiology

Robert-Koch-Str. 8

89081 Ulm, Germany

Email: olaf.grebe@medizin.uni-ulm.de 